\title{
Stochastic relation between anomalous propagation in the line-of-sight VHF radio band and occurrences of earthquakes
}

\author{
K. Motojima and N. Haga \\ Faculty of Science and Technology Gunma University, Kiryu Gunma, Japan \\ Correspondence to: K. Motojima (motojima@el.gunma-u.ac.jp)
}

Received: 22 March 2013 - Published in Nat. Hazards Earth Syst. Sci. Discuss.: 29 November 2013

Revised: - Accepted: 10 July 2014 - Published: 19 August 2014

\begin{abstract}
This paper was intended to find out any relation between anomalous line-of-sight propagation on the very high frequency (VHF) band and occurrences of earthquakes near the VHF propagation paths. The television and FM radio broadcasting waves on the VHF band were monitored continuously over the long term. For that purpose, a multidirectional VHF band monitoring system was established and utilized. Anomalous line-of-sight propagation on the VHF band was distinguished from the monitored wave by using a statistical analysis. After the stochastic consideration, it was found out that earthquakes associated with anomalous propagation were characterized by magnitude of earthquakes $M \geq 4.5$, and distances from epicenters $L \leq 75 \mathrm{~km}$. The anomalous propagation was monitored on the VHF band a few days before the associated earthquakes occurred. Moreover, the anomaly appeared on multidirectional propagation paths simultaneously. The anomaly on the line-of-sight propagation indicates the possibility of narrowly focusing the area of the epicenter of earthquake.
\end{abstract}

\section{Introduction}

Short-term earthquake prediction is one of the most important research tasks for disaster prevention in a country with frequent earthquakes like Japan. Many geophysical electromagnetic phenomena associated with seismicity have been reported, and most reports can be classified into two groups: direct or indirect observations. As one of the direct observations, low-frequency electromagnetic emissions from the earthquake hypocenter were measured for earthquake predictions (Smith et al., 1990; Hayakawa et al., 1996). The observations indicate that the background noise had been in- creased a few weeks prior to the corresponding earthquake (Gokhberg et al., 1982), and these ultra low frequency (ULF) noises might have resulted from microfracturing progression in the lithosphere. As for indirect observations, anomalous propagation in very low frequency (VLF) or upper band was suggested as a promising candidate for earthquake prediction, because any disturbance at the bottom of ionosphere causes anomalous propagation of the radio waves. Remarkable data have been reported on VLF Omega propagation signal from Tsushima, Japan, to Inubo, Japan, in January 1995. The phenomenon was the signal amplitude (and/or phase) variations around sunrise and sunset times and appeared a few days prior to the Kobe, Japan, earthquake which occurred on 17 January 1995. The anomalous propagation data can be explained in terms of fall of ionospheric bottom (Molchanov and Hayakawa, 1998; Hayakawa et al., 2010).

In the meantime, some researchers observed the FM radio waves on the very high frequency (VHF) band in Japan. They reported that anomalous propagation from over-horizon FM transmitter signals was observed. The anomaly seemed to be associated with earthquakes (Yasuda et al., 2009). Other researchers inferred that the anomalous propagation was influenced by the perturbation in the troposphere. They considered that the perturbed region was within a radius of $100 \mathrm{~km}$ from the epicenters of earthquakes (Yonaiguchi et al., 2007).

The purpose of this paper is to find out any relation between anomalous line-of-sight propagation on the VHF band and occurrences of earthquakes. Waves from FM radio and TV broadcasting stations within the line-of-sight region had been observed continuously over the long term. The main target of transmitting stations was placed at Tokyo in Japan. An observation point has been set at Kiryu, which is located about $90 \mathrm{~km}$ north of Tokyo. Therefore, the observation point 


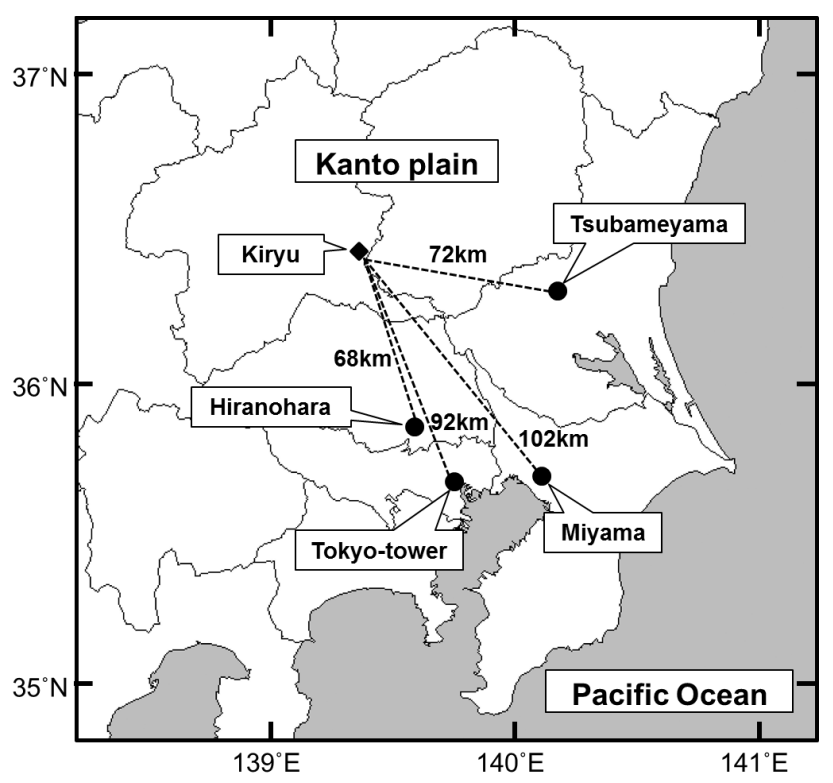

Figure 1. The Kanto plain in Japan, the locations of transmitter stations (FM radio and TV broadcasting: solid circles), observational point (Kiryu: solid diamond) and the propagation paths (dashed lines).

is placed near the outer edge of the line-of-sight range, not over the horizon. To find out the relation between the anomalous propagation and seismicity, we adopted statistical analysis to the observational results and calculated the probability gain, which is the ratio of the observational probability to the non-related probability. These are further distinguished from the previous reports (Fujiwara et al., 2004; Yonaiguchi et al., 2007).

\section{Multidirectional VHF band monitoring system}

The purpose of this paper is to find out any relation between anomalous line-of-sight propagation on the VHF band and occurrences of earthquakes near the propagation paths. We intended to explore the relation, so that a multidirectional VHF band monitoring system was established. The original feature of the monitoring system is a line-of-sight observation method in which the VHF radio band propagation from multidirectional line-of-sight broadcast stations is monitored. The monitoring system is placed in Kiryu, Japan $\left(36^{\circ} 25^{\prime} 26^{\prime \prime} \mathrm{N}, 139^{\circ} 20^{\prime} 58^{\prime \prime} \mathrm{E}\right)$, which is located about $90 \mathrm{~km}$ north of Tokyo. Map positions of Kiryu, Tokyo and the FM radio and TV broadcasting stations are described in Fig. 1. The path lengths from each target broadcasting station to monitoring point (Kiryu), station names and broadcasting frequencies are listed in Table 1.

In the troposphere the refractive index is known to affect the line-of-sight propagation on the VHF radio wave directly. The range of line-of-sight propagation can be estimated by

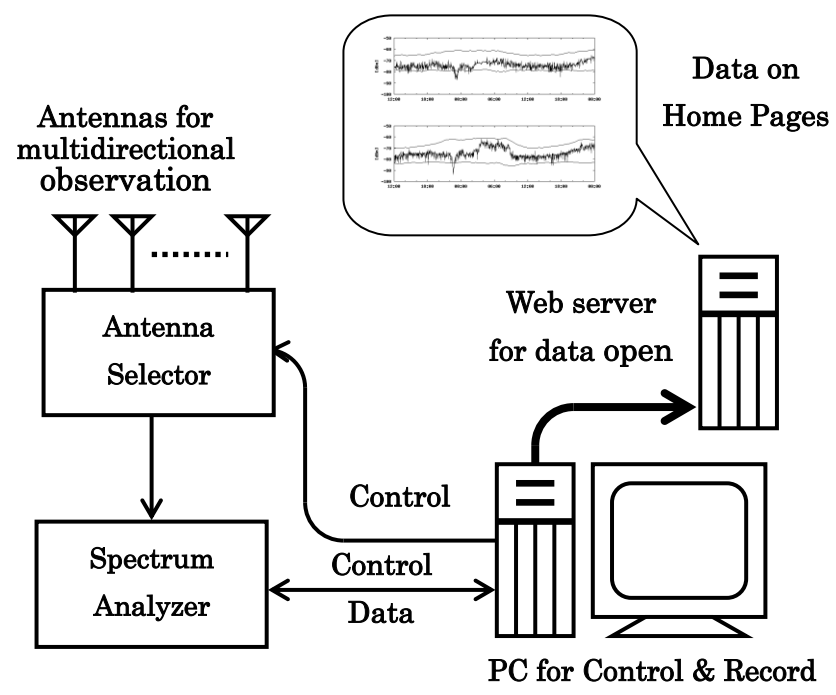

Figure 2. Schematic diagram of the multidirectional VHF band monitoring system.

using following equation:

$d=\sqrt{2 \mathrm{kR}}\left(\sqrt{h_{\mathrm{TX}}}+\sqrt{h_{\mathrm{RX}}}\right)$,

where $d$ is the range scale of line of sight from the transmitting point, and $\mathrm{kR}$ is the effective Earth radius. The normal atmosphere has the coefficient of effective Earth radius, $k=4 / 3$. The $h_{\mathrm{TX}}$ and $h_{\mathrm{RX}}$ are the height of transmitting and receiving antennas above sea level, respectively.

Kiryu is located near the outer edges of the line-of-sight ranges from transmitting stations. Therefore, the refractive index has affected the strength of received waves directly. It means that the receiving point in Kiryu has high sensitivity to anomalous propagation in the troposphere.

For long-term observation an automatic and around-theclock monitoring system consists of multiple antennas, an automatic antenna selector, a spectrum analyzer for radio receiver, a PC for monitoring data storage and a web server for data release. In this monitoring system four 5-element Yagi antennas are used for the multidirectional stations. Each antenna is horizontally orientated to each direction: north, south, east and west. They are installed on the roof of a five-story building. The spectrum analyzer has the role of receiver, which required a wideband and high sensitivity monitor. The automatic antenna selector switches the spectrum analyzer to the antenna oriented to target transmitting station. Data storage PC acquires the receiving signal strength every $30 \mathrm{~s}$. Each raw datum of the received broadcasting wave is graphed and uploaded onto the web server at 30-minute intervals. Then the graphed data can be always visited on the internet. Schematic block diagram of the monitoring system is described in Fig. 2. 
Table 1. Monitored transmitter stations, broadcasting frequencies and path length from each transmitter stations to observation point (Kiryu).

\begin{tabular}{llr}
\hline Transmitter station & Frequency & Path length \\
\hline Tokyo Tower (analog TV signals) & $91.25-217.25 \mathrm{MHz}$ (seven waves) & $92 \mathrm{~km}$ \\
Tokyo Tower (FM radio) & $76.0-80.0 \mathrm{MHz}$ (three waves) & $92 \mathrm{~km}$ \\
Miyama (FM radio) & $78.0-80.7 \mathrm{MHz}$ (two waves) & $102 \mathrm{~km}$ \\
Hiranohara (FM radio) & $85.1 \mathrm{MHz}$ & $68 \mathrm{~km}$ \\
Tsubameyama & $83.2 \mathrm{MHz}$ & $72 \mathrm{~km}$ \\
\hline
\end{tabular}

\section{Anomalous line-of-sight propagation on the VHF band}

In other studies in which the VHF radio waves from overthe-horizon stations were monitored, reception itself means occurrences of anomalous propagation. On the other hand we monitored the line-of-sight propagation wave on the VHF band. Transmitted wave can reach normally into the line-ofsight coverage, so discrimination rule for detection of anomalous propagation was required. For the purpose a certain statistical process was adopted. The data process method is as follows:

1. Received wave has short-time fluctuation and random noise. In order to avoid the influence of them, moving average values (20-minute window) were calculated as observed data.

2. Even the line-of-sight propagation was affected by a diurnal variation on the VHF band. Received waves on the ordinary propagation become weaker in daytime than in nighttime. The reason is that sunlight promotes the atmospheric convection, which decreases the difference in atmospheric refractive index between the surface and the upper air. In order to reduce the influence of diurnal variation, a statistical analysis was performed separately for each specific time slot in a day. A day was divided into 72 time slots: each one has a 20-minute period for example, 0:00-0:20, 0:20-0:40, 0:40-1:00, and so on. Mean values $(m)$ and standard deviations $(\sigma)$ of observed data were separately calculated for each time slot through the observation period.

3. In order to distinguish anomalous observed data from normal ones, we considered the data exceeded beyond $m \pm 3 \sigma$ as the anomalous data. If strength of the propagated wave follows a normal distribution, the probability of exceeding beyond $3 \sigma$ from the mean value $m$ is about $0.27 \%$. It was confirmed through the observation period that the reception data in $\mathrm{dBm}$ for each wave had the normal distribution approximately. When the anomalous data over the $m \pm 3 \sigma$ lasted $30 \mathrm{~min}$ or more, we recognized that the anomalous propagation occurred.

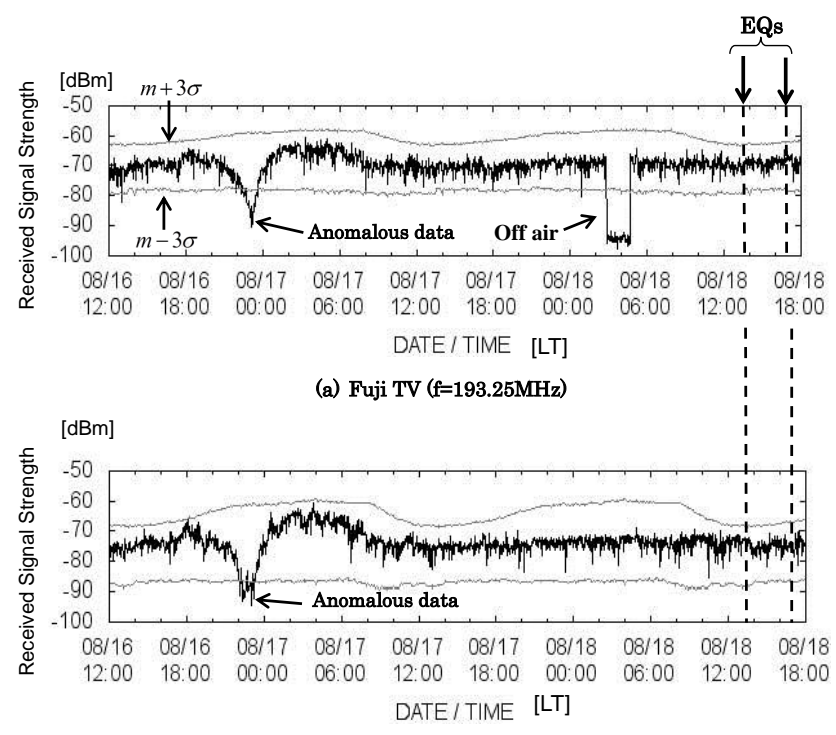

(b) TV Asahi ( $\mathrm{f}=205.25 \mathrm{MHz})$

Figure 3. Temporal evolution of the anomalous propagation on the VHF TV broadcasting band. Two earthquakes associated with the anomaly occurred at 13:36 and 16:55 LT on 18 August 2007, with magnitudes 4.5 and 5.2. The epicenters were $35^{\circ} 21^{\prime} \mathrm{N}, 140^{\circ} 21^{\prime} \mathrm{E}$ and $35^{\circ} 02^{\prime} \mathrm{N}, 140^{\circ} 02^{\prime} \mathrm{E}$, respectively.

Using above process for received wave, we could discriminate the anomalous propagation from normal ones in the lineof-sight propagation on the VHF band.

A temporal evolution, which included an anomalous propagation, is shown in Fig. 3; the upper and lower panels are the received analog TV signal strength on the VHF band, $f=193.25 \mathrm{MHz}, 205.25 \mathrm{MHz}$, respectively. In the upper panel, the anomaly appeared at midnight on 16 August 2007; maximum dip below the $m-6 \sigma$ level occurred at 23:15 LT. Such a low signal strength remained a rare event. In the lower panel, the same anomaly occurred simultaneously at a different frequency. About $40 \mathrm{~h}$ later the associated two earthquakes occurred at 13:36 and 16:55 LT on 18 August with magnitudes 4.5 and 5.2. Both were centered within $75 \mathrm{~km}$ from the propagation path, and times of occurrences are indicated in dashed lines. In Fig. 3 the $m \pm 3 \sigma$ lines are drawn by using 5 -minute time slots to smooth jagged lines. 


\section{Relationship between anomalous propagation and occurrences of earthquakes}

The waves of analog TV video signals on the VHF band had been measured for 1155 days starting from 1 February 2007 to 31 March 2010. Now, they had gone off-air because of migrating over to digital broadcast on ultra high frequency (UHF) band. The multidirectional observation on the FM broadcast waves started 8 October 2010; it is ongoing observation.

We defined the successive occurrence of anomalous propagation and earthquake generation within a short period of time as "occurrence of anomalous propagation associated with earthquake". However, even if there were no relation between the anomalous propagation and earthquake, both just happened to occur on a short term. Therefore, we have to estimate the unrelated probability $P_{\text {unrel }}\left(t_{\text {per }}\right)$ of the successive occurrences between the occurrence of anomalous propagation and earthquake under no relation. The probability can be derived as described below.

Let us consider that only one anomaly and only one earthquake occur under no relation during the entire observation period, $T_{\text {all }}$. When earthquake occurs within a defined length of time period $t_{\text {per }}$ after the anomaly, we consider that the earthquake is associated with the anomaly.

At first, we derive a probability of no sequential occurrence of both in the defined time period $t_{\text {per }}$, $\left.\bar{P}_{\text {unrel }}\left(t_{\text {per }}\right)\right|_{\text {Neq=1 }}$. It is the probability of the occurrence of the earthquake at a complementary time period, as in the following equation.

$\left.\bar{P}_{\text {unrel }}\left(t_{\text {per }}\right)\right|_{\text {Neq=1 }}=\frac{T_{\text {all }}-t_{\text {per }}}{T_{\text {all }}}$,

where $t_{\text {per }}$ is the defined length of the time-period-correlated anomaly and earthquake, while $T_{\text {all }}$ is the amount of observation time ( $T_{\mathrm{all}}=1155$ days for analog TV wave observation).

When the number of earthquakes which occur out of $t_{\text {per }}$ is $N_{\text {eq }}$, the probability can be obtained via the next equation. Because it is the conditional probability.

$$
\left.\bar{P}_{\text {unrel }}\left(t_{\text {per }}\right)\right|_{\text {Neq }}=\left(\frac{T_{\text {all }}-t_{\text {per }}}{T_{\text {all }}}\right)^{\text {Neq }} \text {, }
$$

where $N_{\text {eq }}$ is the number of earthquake occurrences during the whole observation period.

The event in which the anomaly and earthquakes just happen to occur in a defined time period $t_{\text {per }}$ is a complementary event of $\left.\bar{P}_{\text {unrel }}\left(t_{\text {per }}\right)\right|_{\text {Neq }}$. Therefore, the unrelated probability $P_{\text {unrel }}\left(t_{\mathrm{per}}\right)$ of the sequential occurrence of the anomaly and earthquakes can be obtained as follows:

$P_{\text {unrel }}\left(t_{\text {per }}\right)=1-\left.\bar{P}_{\text {unrel }}\left(t_{\text {per }}\right)\right|_{\text {Neq }}=1-\left(\frac{T_{\text {all }}-t_{\text {per }}}{T_{\text {all }}}\right)^{N_{\text {eq }}}$.

Accordingly, the unrelated probability $P_{\text {unrel }}\left(t_{\text {per }}\right)$ depends on the defined length of time $t_{\text {per }}$.
Table 2. Probability gain PG with respect to the magnitude of earthquakes.

\begin{tabular}{crcccc}
\hline Magnitude $M$ & $N_{\text {eq }}$ & $P_{\text {unrel }}$ & $N_{\text {obs }}$ & $P_{\text {obs }}$ & PG \\
\hline$M \geq 3.0$ & 109 & $1.72 \times 10^{-1}$ & 8 & $2.42 \times 10^{-1}$ & 1.41 \\
$M \geq 3.5$ & 50 & $8.30 \times 10^{-2}$ & 6 & $1.82 \times 10^{-1}$ & 2.19 \\
$M \geq 4.0$ & 35 & $5.89 \times 10^{-2}$ & 6 & $1.82 \times 10^{-1}$ & 3.09 \\
$M \geq 4.5$ & 10 & $1.72 \times 10^{-2}$ & 4 & $1.21 \times 10^{-1}$ & 7.03 \\
$M \geq 5.0$ & 2 & $3.46 \times 10^{-3}$ & 1 & $3.03 \times 10^{-2}$ & 8.76
\end{tabular}

On the other hand, the observational probability $P_{\text {obs }}\left(t_{\text {per }}\right)$ of occurrence of anomalous propagation associated with earthquake can be obtained from the observation result. It is based on real events and is calculated by the following equation:

$P_{\text {obs }}\left(t_{\text {per }}\right)=\frac{N_{\text {obs }}\left(t_{\text {per }}\right)}{N_{\text {anom }}}$,

where $N_{\text {obs }}\left(t_{\text {per }}\right)$ is the number of occurrences of anomalous propagation associated with earthquakes in the length of time period $t_{\mathrm{per}}$, and $N_{\mathrm{anom}}$ is the number of occurrences of anomalous propagation during the whole observation pe$\operatorname{riod} T_{\text {all }}\left(N_{\text {anom }}=33\right.$ on the analog TV video wave). If the observational probability $P_{\mathrm{obs}}\left(t_{\mathrm{per}}\right)$ is close to the unrelated probability $P_{\text {unrel }}\left(t_{\text {per }}\right)$, it means that there may be no relation between the anomalous propagation and earthquake.

The number of occurrences of anomalous propagation associated with earthquakes $N_{\text {obs }}\left(t_{\text {per }}\right)$ depends on the length of time period $t_{\mathrm{per}}$, because the longer time period $t_{\mathrm{per}}$ makes the more anomalous propagation identified as "occurrence of anomalous propagation associated with earthquake". In addition, we propose a new concept of the probability gain PG $\left(t_{\text {per }}\right)$ for estimating the relationship between the anomalous propagation and earthquake. The probability gain PG $\left(t_{\mathrm{per}}\right)$ is the ratio of the observational probability $P_{\text {obs }}\left(t_{\text {per }}\right)$ to the unrelated probability $P_{\text {unrel }}\left(t_{\text {per }}\right)$. It can be obtained as follows:

PG $\left(t_{\text {per }}\right)=\frac{P_{\text {obs }}\left(t_{\text {per }}\right)}{P_{\text {unrel }}\left(t_{\text {per }}\right)}$

If the PG $\left(t_{\text {per }}\right)$ is close to one, it means that there may be no relation between the anomalous propagation and earthquake.

We estimated the probability gains $\mathrm{PG}\left(t_{\mathrm{per}}\right)$ for the varied time period $t_{\text {per }}$ carefully. As the result of it, we reached a conclusion that the probability gain PG $\left(t_{\text {per }}\right)$ became maximum value at the length of time period $t_{\mathrm{per}}=2$ days. In the following subsections the probability gains $\mathrm{PG}\left(t_{\mathrm{per}}=\right.$ 2 days) are shown with respect to magnitude of earthquakes and distances between epicenters and propagation path. 


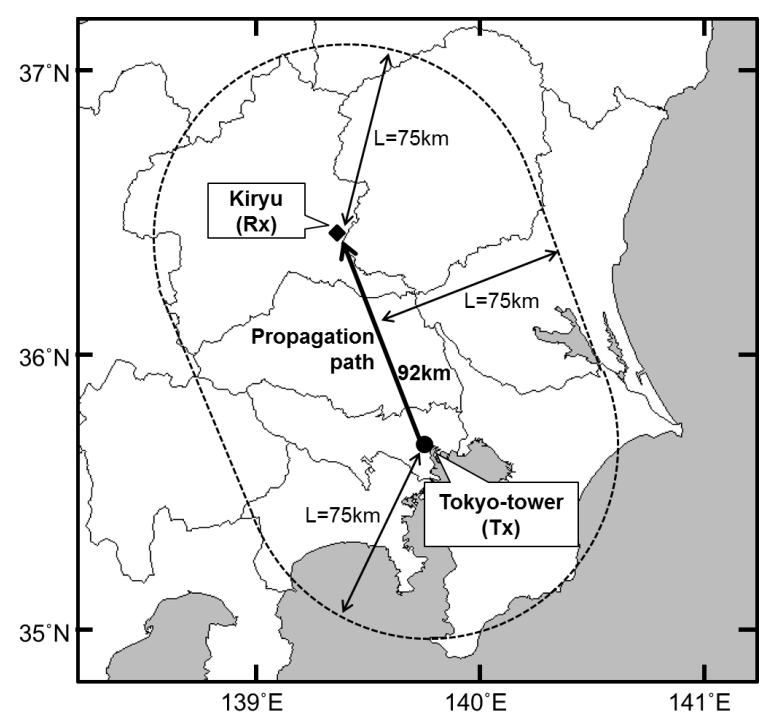

Figure 4. Equidistant curve (dashed curve, $L=75 \mathrm{~km}$ ) from the propagation path of Tokyo Tower to Kiryu (black arrow). Solid circle and diamond indicate the locations of transmitter stations (Tokyo Tower) and the observational point (Kiryu), respectively.

Table 3. Probability gain PG with respect to distances of epicenters to the propagation path.

\begin{tabular}{cccccc}
\hline Distance $L$ & $N_{\text {eq }}$ & $P_{\text {unrel }}$ & $N_{\text {obs }}$ & $P_{\text {obs }}$ & PG \\
\hline$L \leq 200 \mathrm{~km}$ & 54 & $8.93 \times 10^{-2}$ & 7 & $2.12 \times 10^{-1}$ & 2.37 \\
$L \leq 150 \mathrm{~km}$ & 43 & $7.18 \times 10^{-2}$ & 5 & $1.52 \times 10^{-1}$ & 2.12 \\
$L \leq 100 \mathrm{~km}$ & 22 & $3.74 \times 10^{-2}$ & 5 & $1.52 \times 10^{-1}$ & 4.06 \\
$L \leq 75 \mathrm{~km}$ & 10 & $1.72 \times 10^{-2}$ & 4 & $1.21 \times 10^{-1}$ & 7.03 \\
\hline
\end{tabular}

\subsection{Probability gain PG with respect to the magnitude of earthquakes}

Table 2 shows the relation between magnitude of earthquakes and the probability gain PG $\left(t_{\mathrm{per}}=2\right.$ days $)$. The result indicates that the larger magnitude of earthquake was more associated with the anomalous propagation. Especially when the magnitudes of earthquakes were larger than 4.5 , the number of earthquakes was $N_{\mathrm{obs}}=4$. Therefore PG was 7.03 , and they had a strong relation with anomalous propagation. On the other hand, no earthquake happened before anomalous VHF propagation occurrences for the same $t_{\text {per }}$.

\subsection{Probability gain PG with respect to distances of epicenters to the propagation path}

We considered the positional relation between the epicenter location and the propagation path. Therefore, the perpendicular distance from epicenters to the propagation path was defined as a distance $L$. Equidistant curve from the propagation path of Tokyo Tower to Kiryu is shown as an example of $L=75 \mathrm{~km}$ in Fig. 4 .

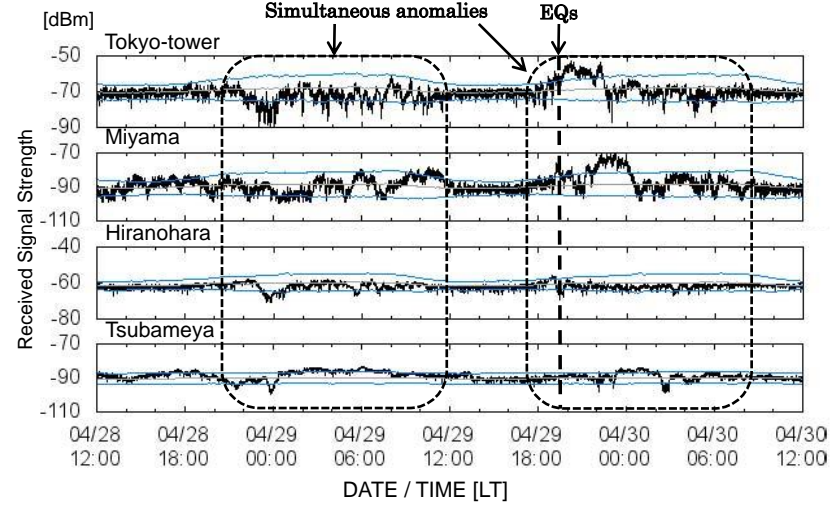

Figure 5. Temporal evolution on 28 to 30 April 2012. The dashed line indicates the earthquake associated with the anomaly that occurred at 19:28 LT on 29 April 2012, 5.8 magnitude, with epicenter at $35^{\circ} 42^{\prime} \mathrm{N}, 140^{\circ} 36^{\prime} \mathrm{E}$.

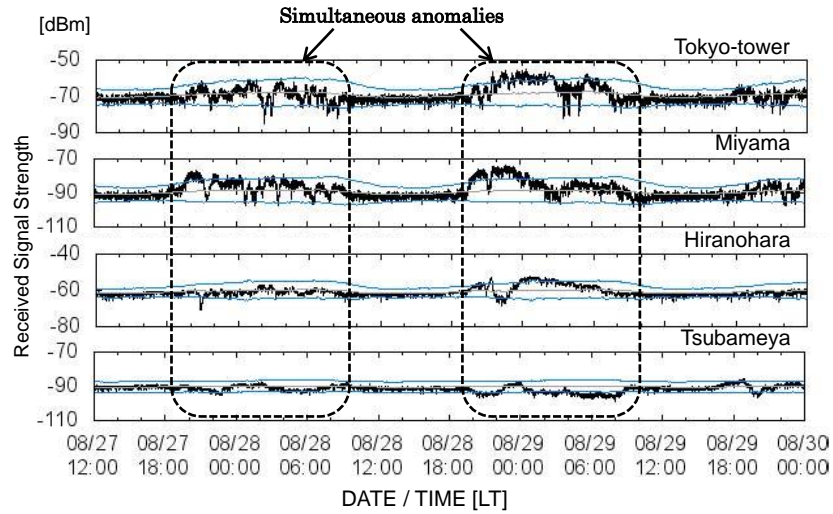

Figure 6. Temporal evolution on 27 to 30 August 2011. The earthquake associated with the anomaly that occurred at 18:32 LT on 31 August 2011, 4.6 magnitude, with epicenter at $35^{\circ} 33^{\prime} \mathrm{N}$, $140^{\circ} 05^{\prime} \mathrm{E}$ in northern Tokyo Bay.

Table 3 shows the relation between the distance $L$ and the probability gain $\mathrm{PG}\left(t_{\mathrm{per}}=2\right.$ days $)$. The result indicates that the closer epicenters to the propagation path were more associated with the anomalous propagation. Earthquakes about $150 \mathrm{~km}$ away from the propagation path had little relation to the anomalous propagation.

\subsection{Synchronism detection of anomalous propagation in the multidirectional FM broadcasting waves}

Figures 5 and 6 are the observational result in the multidirectional FM waves, which were incoming from four different transmitting stations - Tokyo Tower, Miyama, Hiranohara and Tsubameyama stations - as shown in Fig. 1. Black solid lines indicate the received signal strengths. Three gray lines mean an upper limit of ordinary propagation $(m+3 \sigma)$, mean value $(m)$ and a lower limit of it $(m-3 \sigma)$ in order 
from top. They were statistically derived from the long-term observational data.

Figure 5 shows the temporal evolutions on 28 to 30 April 2012. The received signal strengths from Tokyo Tower and Miyama station had two obvious anomalies that occurred almost at the same time: from 10 p.m. on 28 April to 11 a.m. on 29 April, and from 7 p.m. on 29 April to 6 a.m. on 30 April. Both anomalies lasted half a day. The other two waves from Hiranohara and Tsubameyama stations had short-time anomaly at 23:30 LT on 28 April. During the second simultaneous anomalies, an earthquake occurred at 19:28 LT on 29 April 2012, 5.8 magnitude, epicenter location $35^{\circ} 42^{\prime} \mathrm{N}, 140^{\circ} 36^{\prime} \mathrm{E}$. The anomalous propagation on above data seemed to be a precursor of the earthquake.

Figure 6 shows another anomalous propagation which occurred on 27 to 29 August 2011. The received signal strengths from Tokyo Tower and Miyama station had obvious anomalies. And the waves from Hiranohara and Tsubameyama stations had weak anomalies. It indicated a similar tendency of the observation in Fig. 5. An earthquake happened subsequent to this anomaly several days later. It occurred at 18:32 LT on 31 August 2011, 4.6 magnitude earthquake, centered in northern Tokyo Bay $\left(35^{\circ} 33^{\prime} \mathrm{N}\right.$, $140^{\circ} 05^{\prime} \mathrm{E}$ ). The anomalous propagation may be the precursor of the earthquake.

Moreover, we had considered the relation between the anomalous propagation and the atmospheric phenomena. Until now, we had no clear statistical results which indicate existence of the relation between both. However, we have noticed an empirical relation between anomalous propagation and surface wind velocity near the propagation path. When the wind velocity was $3 \mathrm{~m} \mathrm{~s}^{-1}$ or more on the propagation path, anomalous propagation was not monitored at all. Although an anomalous propagation happened to appear under no wind condition, it disappeared with increasing the wind velocity.

\section{Summary}

In this paper, the relation between anomalous line-of-sight propagation on the VHF band and occurrences of earthquakes was investigated by using the statistical analysis. For that purpose, the multidirectional VHF band monitoring system was established, and the line-of-sight VHF waves had been monitored on the long term. In order to estimate the relation between the two, the new conception of the probability gain was introduced. As the results of observation for over 3 years and the stochastic consideration, we found out the relation between anomalous line-of-sight propagation and earthquakes. Especially earthquakes associated with anomalous propagation were characterized by magnitude of earthquakes $M \geq 4.5$, and distances from epicenters $L \leq 75 \mathrm{~km}$. The event probability of the line-of-sight propagation increased just a few days prior to earthquakes cate- gorized by the above. Moreover, synchronism detection of anomalous propagation in the multidirectional FM broadcasting waves was shown. The anomalies associated with earthquakes sometimes occurred almost at the same time on the plural radio waves via different pathways. These phenomena have the possibility of narrowly focusing the area of the epicenter of the earthquake.

The important relation between the two could be found, but it is not yet ready to be accepted as a fact. Additionally, we have to elucidate the mechanism between the anomalous propagation and occurrences of earthquakes. These are considered to be our future works, and so the observation and the analysis should be continued extensively in the future as well.

Edited by: R. Lasaponara

Reviewed by: M. Dubrov and two anonymous referees

\section{References}

Fujiwara, H., Kamogara, M., Ikeda, M., Liu, J.Y., Sakata, H., Chen, Y. I., Ofuruton, H., Muramatsu, S., Chuo, Y. J., and Ohtsuki, Y. H.: Atmospheric anomalies observed during earthquake occurrences, Geophys. Res. Lett., 31, L17110, doi:10.1029/2004GL019865, 2004.

Gokhberg, M. B., Morgounov, V. A., Yoshino, T., and Tomizawa, I.: Experimental measurement of electromagnetic emissions possibly related to earthquakes in Japan, J. Geophys. Res., 87, 7824$7828,1982$.

Hayakawa, M., Kawate, R., Molchanov, O. A., and Yumoto, K.: Results of ultra-low-frequency magnetic field measurements during the Guam earthquake of 8 August 1993, Geophys. Res. Lett., 23, 241-244, doi:10.1029/95GL02863, 1996.

Hayakawa, M., Kasahara, Y., Nakamura, T., Muto, F., Horie, T., Maekawa, S., Hobara, Y., Rozhnoi, A. A., Solovieva, M., and Molchanov, O. A.: A statistical study on the correlation between lower ionospheric perturbations as seen by subionospheric VLF/LF propagation and earthquakes, J. Geophys. Res., 115, A09305, doi:10.1029/2009JA015143, 2010.

Molchanov, O. A. and Hayakawa, M.: Subionospheric VLF signal perturbations possibly related to earthquakes, J. Geophys. Res., 103, 17489-17504, doi:10.1029/98JA00999, 1998.

Smith, A. C. F., Bernardi, A., McGill, P. R., Ladd, M. E., Helliwell, R. A., and Villard, O. G. Jr.: Low-frequency magnetic field measurements near the epicenter of the Ms 7.1 Loma Prieta earthquake, Geophys. Res. Lett., 17, 1465-1468, doi:10.1029/GL017i009p01465, 1990.

Yasuda, Y., Ida, Y., Goto, T., and Hayakawa, M.: Interferometric direction finding of over-horizon VHF transmitter signals and natural VHF radio emissions possibly associated with earthquakes, Radio Sci., 44, RS2009, doi:10.1029/2008RS003884, 2009.

Yonaiguchi, N., Ida, Y., and Hayakawa, M.: On the statistical correlation of over-horizon VHF signals with meteorological radio ducting and seismicity, J. Atmos. Sol-terr. Phys., 69, 661-674, doi:10.1016/j.jastp.2007.01.007, 2007. 